\title{
Cirugía radical del cáncer de cérvix por laparoscopia. Reporte de un caso y revisión de la literatura
}

\author{
Mario Arturo González Mariño*; Byron Cardoso Medina**
}

\section{RESUMEN}

Se presenta el primer caso de cirugía radical laparoscópica por cáncer de cérvix en el Hospital Central de la Policía Nacional, Santafé de Bogotá, Colombia, se hace una aproximación al enfoque económico del uso de esta tecnología en el manejo de la histerectomía por patología benigna y maligna y se exponen sus potenciales ventajas .

Se incluye información sobre número de ganglios resecados y complicaciones de la cirugía con base en reportes sobre esta técnica quirúrgica.

Conclusiones: La cirugía laparoscópica en el cáncer de cérvix constituye un enfoque terapéutico importante que en estudios descriptivos ha mostrado ofrecer ventajas que en algunos aspectos parecen superar la realización de la cirugía convencional. Se requiere sin embargo de estudios experimentales que ubiquen su valor en el tratamiento del cáncer de cérvix.

PALABRAS CLAVES: Cáncer de cérvix, laparoscopia, histerectomía radical.

\section{SUMMARY}

We report the first case of radical surgery by laparoscopy for the treatment of cervical cancer performed at the Central Hospital of National Police, Santafé de Bogotá, Colombia. An economical analysis on this technique when it is applied to patients who undergo a hysterectomy because of benign or cancer disease is undertaken and the proposed advantages of this surgical procedure are shown.

Information on the number of lymphatic nodes resected and surgical complications are reported on the basis of related papers.

Conclusions: The laparoscopic surgery for the treatment of cervical cancer is an important therapeutic technique which in descriptive studies has shown to have some advantages over the conventional approach. Experimental design studies to clear the position of this method on the therapeutic procedures are highly recommended.

KEY WORDS: Cervical cancer, laparoscopy, radical hysterectomy.

\section{Introducción}

El desarrollo de la cirugía laparoscópica la ha convertido en un procedimiento alternativo en las patologías neoplásicas por permitir una adecuada visualización del campo quirúrgico, tener mejor resultado estético, facilitar un regreso laboral mas pronto y en mejores condiciones y presentar una menor tasa de infección haciendo del procedimiento una experiencia más amigable para la paciente (1).

Uno de los usos propuestos es en el tratamiento del cáncer de cérvix. Con la laparoscopia se magnifican las

\footnotetext{
* Médico, Ginecología Oncológica y Endoscopia Ginécológica

** Médico, Ginecología-Obstetricia y Endoscopia Ginecológica. Instructor de Endoscopia Ginecológica, Hospital Central Policia Nacional. Santafé de Bogotá.
}

estructuras entre 5 y 7 veces, se puede hacer una adecuada hemostasia y el tejido tumoral puede ser visualizado adecuadamente (2).

Con base en el primer caso de cirugía radical laparoscópica realizado en el Hospital Central de la Policía (HOCEN), Santafé de Bogotá se hace un análisis sobre esta técnica.

\section{Caso clínico}

Paciente de 34 años G 3 P2 A1, peso: $60 \mathrm{Kg}$ y Talla: $160 \mathrm{~cm}$, con diagnóstico por biopsia de carcinoma escamocelular no queratinizante e infiltrante de cérvix, sin antecedentes quirúrgicos ni médicos de importancia. Se clasificó como estado IB1.

El procedimiento quirúrgico de acuerdo con los protocolos del Hospital Central de la Policía Nacional fue 
Histerectomía radical tipo III, linfadenectomía pélvica y paraaórtica desde la arteria mesentérica inferior y ooforopexia (3).

\section{Procedimiento laparoscópico:}

Se colocó la paciente en posición de litotomía modificada con la menor flexión posible de los muslos sobre el abdomen. Luego de la preparación del área quirúrgica, se realizó cateterismo vesical con sonda Foley número 16 y se colocó manipulador uterino con delineador de fornix vaginal.

Con aguja de Verres se insufló $\mathrm{CO} 2$ y se usaron cuatro puertos: dos puertos de 10 milímetros, intraumbilical y a cuatro centímetros por encima del pubis, con válvula o pistón y dos puertos de 5 milímetros en ambas fosas ilíacas por fuera de los vasos epigástricos inferiores y un poco más alto de lo usual para tener un mejor acceso a la histerectomía.

Con la paciente en posición de Trendelenburg de 20 a 30 grados, mediante el telescopio de 10 milímetros, 0 grados, en el puerto intraumbilical se valoró la cavidad abdominal.

Luego de colocar los puertos accesorios se procedió a cambiar la localización del telescopio al puerto suprapúbico. Con "grasper" a través del puerto central se rechazaron las asas, se levantó el peritoneo con dos pinzas y con ayuda de hidrodisección se incidió el peritoneo a nivel de la bifurcación aórtica en dirección cefálica hasta evidenciar la arteria mesentérica inferior.

Mediante disección roma y cortante con tijera de Metzembaum, se procedió a realizar la disección de los ganglios paraórticos izquierdos, ganglios retroaórticos, preaórticos, interaortocavos, precavos, paracavos derechos y retrocavos (Figura 1). Se continuó con ilíacos comunes, extrayendo progresivamente el tejido ganglionar por uno de los puertos de 10 milímetros, con reductores, por ser el instrumental de $5 \mathrm{~mm}$.

La hemostasia y el control de la posible salida de linfa se realizó con fórceps de microbipolar y en caso de superficie cruenta se realizó taponamiento con sustancias hemostásicas ("gelfoam" o "surgicel").

El telescopio se trasladó al puerto intraumbilical, se seccionaron los ligamentos redondo y útero ovárico derecho, disecando el ligamento infundíbulo-pélvico y separando el uréter (Figura 2).

Se ingresó en el espacio pararectal, se expuso la arteria y vena ilíacas externas y se realizó disección de los ganglios hasta la vena circunfleja ilíaca. Se identificó el nervio obturador, arteria ilíaca interna y su rama vesical superior y se efectuó disección de sus ganglios (Figura 3).

Se localizó la arteria uterina a su salida de la hipogástrica, se realizó coagulación con fórceps de microbipolar, se colocaron clips proximales y distales al sitio donde se seccionaría, y se liberó (Figura 4).

Igual procedimiento se completó en el lado izquierdo.
En el techo del túnel del uréter se colocaron dos clips y se incidió siguiendo el uréter hasta su ingreso a la pared vesical.

Se colocó el útero en anteroversoflexión, se identificaron ligamentos uterosacros, se incidió el peritoneo encima y por debajo de ellos, y se realizó coagulación y corte cerca de la pelvis. Rechazando el útero en sentido contrario, se visualizó el ligamento cardinal, el cual se tomó progresivamente, coaguló con fórceps cerca de su inserción pélvica y se cortó, completando la radicalidad.

En el paso de ampliación de la separación vesical previo a la colpotomía, ésta se lesionó, por lo cual se decidió continuar por vía vaginal y reparar la lesión por esta vía.

Se regresó al tiempo laparoscópico, se revisó hemostasia y lavó la cavidad. Se dejó abierto el peritoneo y se realizó ooforopexia a través de túnel retroperitoneal en las goteras parieto-cólicas, marcando los ovarios çon clips.

El tiempo quirúrgico fue de 11 horas, la evolución postoperatoria fue satisfactoria, la hemoglobina disminuyó su valor preoperatorio de 14.9 a 11.9 en el postoperatorio. La vía oral se inició en el primer día postoperatorio siendo bien tolerada, la paciente en este mismo día inició su deambulación, insistiendo de su parte en la salida.

En el tercer día postoperatorio, se autorizó su egreso con sonda vesical.

El reporte de patología mostró bordes de sección libres de tumor. En la linfadenectomía se encontraron 6 ganglios paraaórticos y 40 pélvicos, sin compromiso metastásico.

\section{Discusión}

La cirugía convencional radical de cérvix requiere comúnmente para su realización una incisión que va por encima del ombligo hasta el pubis, esto se relaciona con un mayor riesgo de complicaciones postoperatorias: infección, dehiscencia de herida, dolor, insatisfacción con el resultado estético y un regreso laboral a las actividades regulares más prolongado con repercusiones en la productividad.

La cirugía laparoscópica surge como opción terapéutica que supera estas limitaciones, permitiendo una adecuada radicalidad.

Las ventajas propuestas en general para la histerectomía por laparoscopia implican disminución del dolor, menor tiempo de hospitalización y de recuperación, menores tasas de complicaciones (5-6), así como un menor costo a la comunidad por un retorno mas rápido a la vida activa $(1,7)$.

\section{Consideraciones Económicas}

El beneficio económico de un procedimiento se puede evaluar en general mediante la relación costo-beneficio y costo eficacia. Bajo el primer enfoque se califica en 
Figura $0002 \mathrm{i} 01$

LINFADENECTOMIA PARAAORTICA. SE OBSERVA LA ARTERIA

MESENTERICA INFERIOR, LIMITE SUPERIOR DE LA DISECCIÓN

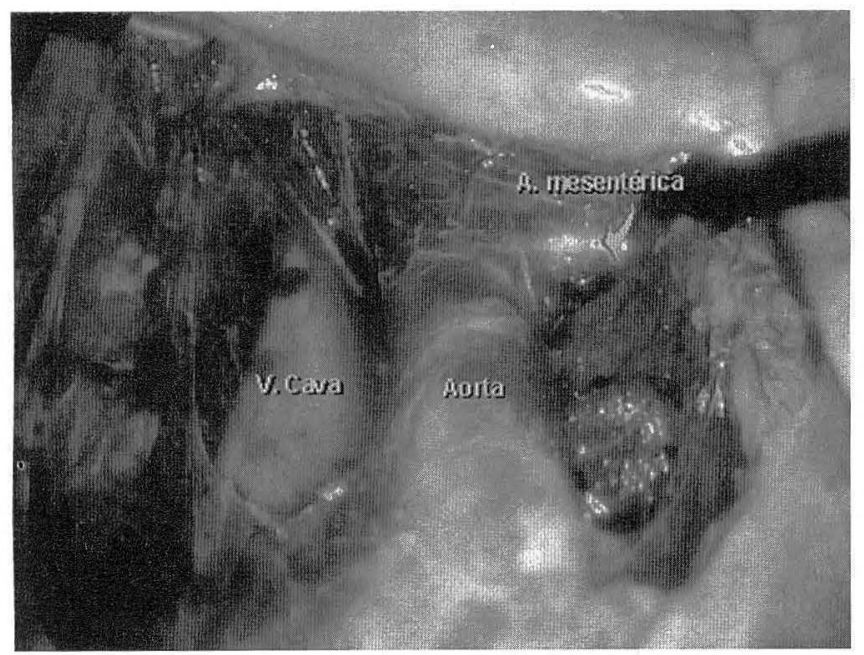

Figura $0002 \mathrm{i} 03$

LINFADENECTOMIA PELVICA

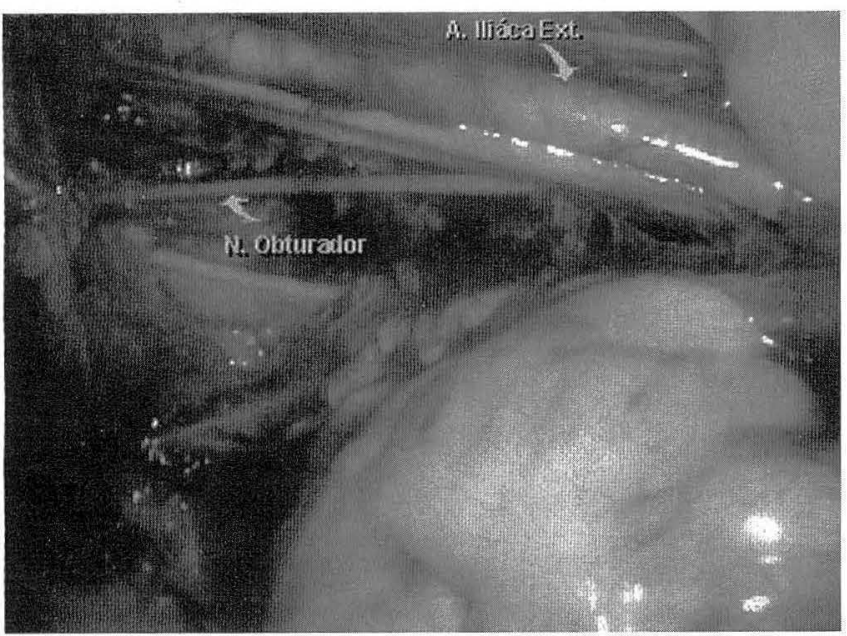

pesos el valor de la vida humana como factor productivo de acuerdo con la edad, posición y capacidad económica de cada individuo. El costo eficacia, en cambio, compara los costos con los beneficios resultantes (8); se puede en este caso, emplear indicadores de morbilidad, mortalidad, recuperación y satisfacción.

En la relación costo-beneficio la laparoscopia constituye una importante ganancia por un regreso laboral en
Figura $0002 \mathrm{i} 02$

LINFADENECTOMIA A NIVEL DE VASOS ILIACOS COMUNES Y DISECCION DEL LIGAMENTO INFUNDIBULO PELVICO DERECHOS. EL URETER INGRESA A LA PELVIS

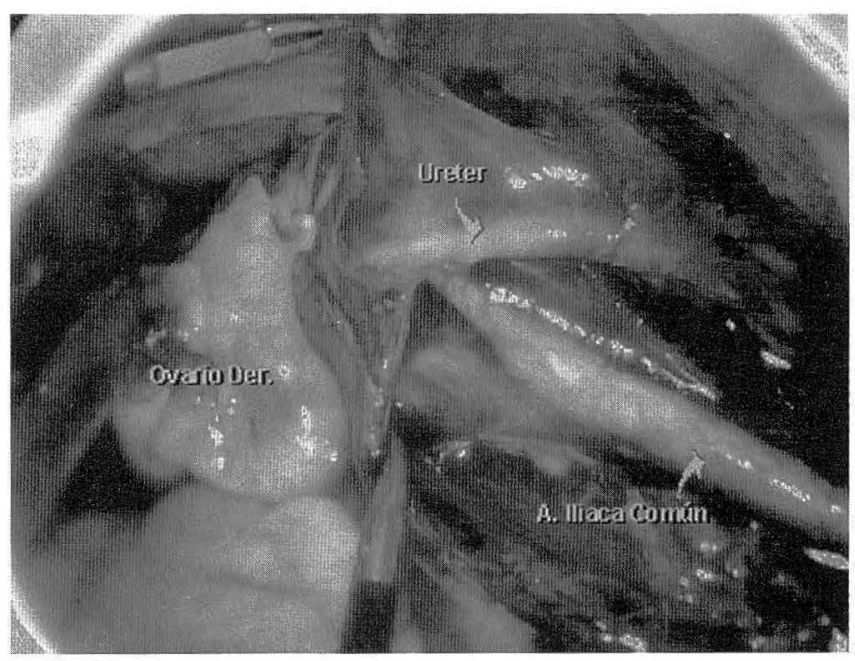

Figura $0002 \mathrm{i} 04$

ARTERIA UTERINA DESPUES DE LA COLOCACION DE CLIPS

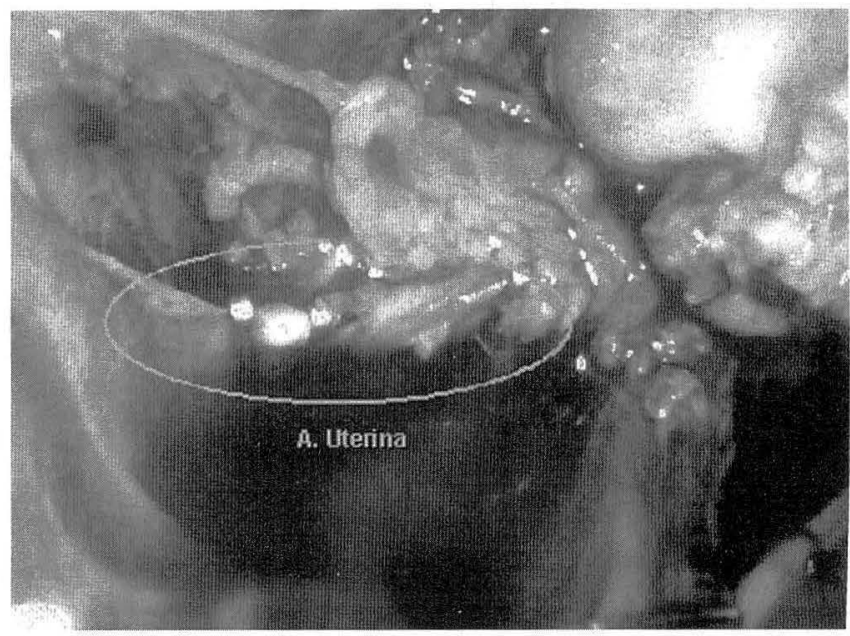

mejores condiciones sin una incisión limitante (al comparar las técnicas de histerectomía, se encuentra que la incisión requerida para la laparotomía es el factor más implicado en una recuperación demorada (9).

En el costo eficacia la laparoscopia copia la cirugía abierta, permite visualización de las estructuras y adecuada radicalidad con menor morbilidad y mayor satisfacción de la paciente. 
Ellström y cols (9), en un estudio en que se aleatorizan las pacientes a histerectomía abdominal o histerectomía total laparoscópica calculan como costos directos los causados durante el período de hospitalización y los indirectos como la pérdida del valor de la producción; se encontró que los costos para el hospital fueron despreciables, mientras que los costos indirectos se redujeron a la mitad con el uso de la laparoscopia.

\section{Ventajas}

Se han asignado una serie de ventajas del uso de la laparoscopia en la cirugía radical sobre el abordaje convencional. Sin embargo estas aseveraciones surgen de estudios observacionales. Tabla 1.

Tabla 1

Ventajas atribuidas a la cirugia radical laparoscopica en el carcinoma de cervix

- MEJOR VISUALIZACION DE LAS

ESTRUCTURAS (2)

- MEJOR RESUltado ESTETICO $(1,12)$

- MENOR SANGRADO

INTRAOPERATORIO (12)

- MEJORIA EN LA ACTIVIDAD

PRODUCTIVA (12)

- DISMINUCION DE ADHERENCIAS $(2,4)$

Número de Ganglios linfáticos

El número de ganglios linfáticos en este caso se compara a los de las series revisadas. Tabla 2.

Tabla 2

Numero de ganglios en pacientes de cirugia radical por cancer de cervix

\begin{tabular}{|lll|}
\hline $\begin{array}{l}\text { Autores y No. de } \\
\text { Pacientes }\end{array}$ & $\begin{array}{c}\text { Promedio } \\
\text { y rango } \\
\text { de ganglios } \\
\text { paraaórticos }\end{array}$ & $\begin{array}{c}\text { Promedio } \\
\text { y rango } \\
\text { de ganglios } \\
\text { pélvicos }\end{array}$ \\
\hline Spirtos y cols (10) (11) & $6.5(4-9)$ & $19.2(12-26)$ \\
Hsieh y cols (10) (12) & $6.6(5-11)$ & $19.2(11-26)$ \\
Schneider (33)(13) & $4.3(0-15)$ & $22.9(10-61)$ \\
HOCEN (1) & 6 & 40 \\
\hline
\end{tabular}

Roy y cols señalan un promedio de 27 ganglios (8-59) (14) en un informe que reporta el número global del conteo ganglionar en 52 pacientes a quienes se realizó linfadenectomía por laparoscopia, pero la histerectomía radical se efectuó por vía abdominal o vaginal de acuerdo con los procedimientos convencionales.

A pesar de reportes de obtención de 52 ganglios pélvicos con la cirugía convencional (15), el muestreo ganglionar es variable aunque se realice la misma técnica quirúrgica, existiendo diferencias en el contenido ganglionar entre las personas y en la forma como los patólogos evalúan y cuentan los ganglios linfáticos. Por lo tanto, se considera que el conteo ganglionar no es comparable de manera válida, planteándose opciones para evaluar la calidad de la linfadenectomía como la filmación o la fotografía (16).

\section{Tiempo Quirúrgico}

El tiempo quirúrgico es un factor que disminuye a medida que se tiene más experiencia y coordinación del equipo quirúrgico (10-11). En histerectomía radical, tipo III , con linfadenectomía pélvica y aórtica la serie de Spirtos y cols (11), reporta las dos primeras cirugías como las más prolongadas, logrando posteriormente tiempos de 3 a 4 horas (pacientes seleccionadas, se usaron endosuturas y coagulador con corriente de argón). Similar tendencia reportan Hsieh y cols (12) con un tiempo promedio de 298,3 minutos y Schneider (12) con 295 minutos (histerectomía radical vaginal asistida por laparoscopia).

\section{Complicaciones intraoperatorias}

En patología benigna, con la histerectomía vaginal asistida por la laparoscopia se reportan $4 \%$ de complicaciones mayores y $1.2 \%$ de necesidad de conversión de laparoscopia en laparotomía (17).

Para la histerectomía radical y linfadenectomía, se reportan las siguientes complicaciones. Tabla 3.

Tabla 3

\section{COMPLICACIONES INTRAOPERATORIAS}

\begin{tabular}{|lcc|}
\hline $\begin{array}{l}\text { Autores y No. } \\
\text { de pacientes }\end{array}$ & $\begin{array}{c}\text { No. de } \\
\text { complicaciones }\end{array}$ & $\begin{array}{c}\text { Tipo de } \\
\text { complicación }\end{array}$ \\
\hline $\begin{array}{l}\text { Spirtos y cols }(10)^{(11)} \\
\text { Hsieh y cols }(10)^{(12)}\end{array}$ & 0 & - \\
Schneider(33) ${ }^{(13)}$ & 1 & $\begin{array}{c}\text { Vena cava } \\
\text { Vena ilíaca } \\
\text { izquierda, } \\
\text { ureteral, } \\
\text { vesical (3) }\end{array}$ \\
HOCEN & 5 & Vesical \\
\hline
\end{tabular}


Tabla 4

COMPLICACIONES POSTOPERATORIAS

\begin{tabular}{|c|c|c|}
\hline $\begin{array}{l}\text { Autores y No. } \\
\text { de pacientes }\end{array}$ & $\begin{array}{c}\text { Total de } \\
\text { complicaciones }\end{array}$ & $\begin{array}{c}\text { Tipo de } \\
\text { complicación } \\
\text { y Número }\end{array}$ \\
\hline Spirtos y cols (10) & 0 & - \\
\hline Hsieh y cols $(10)^{(12)}$ & 1 & - Fiebre \\
\hline Schneider(33) ${ }^{(13)}$ & 12 & $\begin{array}{l}\text { - Infección de } \\
\text { vias urinarias (5) } \\
\text { - Fiebre (4) } \\
\text { - I infedema en } \\
\text { la pierna } \\
\text { izquierda (2) } \\
\text { - Lesión } \\
\text { del nervio } \\
\text { obturador (1) }\end{array}$ \\
\hline
\end{tabular}

Con el mayor uso de la laparoscopia en diferentes neoplasias, se han reportado casos de recurrencia en los sitios de inserción de los trócares y de carcinomatosis peritoneal difusa principalmente en casos de adenocarcinomas, pero también en cánceres escamosos de cérvix (18-19).

\section{Conclusiones}

La cirugía radical laparoscópica ofrece una alternativa a estudiar dentro del abordaje quirúrgico de la Ginecologia Oncológica. Si bien se han planteado beneficios con su uso, estos se hacen con base en estudios descriptivos, pacientes seleccionadas, número pequeño de casos y en centros con la disponibilidad tecnológica y humana para efectuar este procedimiento. Aún se desconoce si las condiciones requeridas para la laparoscopia (ejemplo, insuflación de gas, extracción de tejido tumoral por un mismo sitio o de diámetro pequeño) puedan modificar el comportamiento biológico del tumor en algunas pacien- tes, y de ser esto verídico, si existen factores de riesgo personales o del tumor que permitan excluir a la laparoscopia como abordaje quirúrgico en este grupo.

La adquisición de destreza en cirugia radical laparoscópica representa un reto de gran magnitud para el grupo de cirujanos, el cual debe conseguir una adecuada coordinación, desarrollo técnico y disponer de un sólido conocimiento sobre la patología, anatomía y modalidades terapéuticas disponibles. Por su formación es al Ginecólogo Oncólogo a quien corresponde liderar o conformar este proceso.

Si bien los costos intraoperatorios de la laparoscopia parecen ser mayores, el desempeño posterior de estas pacientes se considera mejor, consiguiendo una mejor aceptación de la enfermedad y un mejor desempeño personal, laboral y social. En general, en la evolución hasta cáncer invasivo de cérvix existen fallas en los sistemas de prevención por lo cual el esfuerzo de compensación a nivel institucional por ofrecer un tratamiento ideal que le brinde a la afectada la posibilidad de integrarse de una mejor manera a la sociedad parecería ser lo menos oneroso. Sin embargo, en la laparoscopia para cáncer de cérvix es el conocimiento actual el que no clarifica su uso como tratamiento de elección siendo necesario el resultado de estudios experimentales, multicéntricos que comparen su empleo con el abordaje quirúrgico convencional. Consideramos que la paciente programada en junta quirúrgica para cirugía radical laparoscópica por cáncer de cérvix debe entender y aceptar que el procedimiento propuesto se encuentra en evaluación por ser una modalidad nueva, que es una alternativa promisoria en el manejo de esta enfermedad y debe ser informada de sus riesgos.

\section{Agradecimientos}

Al Teniente Coronel, Doctor Mauricio Achury por su empeño en lograr que el Departamento de Gineco-Obstetricia del Hospital Central de la Policia Nacional alcance una posición de primer orden a nivel nacional y al Doctor Carlos Anibal Ramos integrante del equipo quirúrgico del caso reportado por su apoyo y colaboración.

\section{BIBLIOGRAFIA}

1. Cravello L.. Montgolfier R de.. DErcole C., Roger V.. Blane B. Endoscopic surgery. The end of classic surgery? European Journal of Obstetrics \& Gynecology and Reproductive Biology 1997; 75 : 103-106.

2. Possover M., Krause N., Kühne-Heid R., Schneider A.Value of laparoscopic evaluation of paraaortic and pelvic lymph nodes for treatment of cervical cancer. Am .l Obstet Gynecol 1998; 178: 806-810.

3. González MA. Cáncer de Cérvix. En González. M.A. Cáncer Ginecológico. Editorial Lerner, Colombia 1999; 9-58.

4. Chen MD.. Teigen GA., Reynolds H., Johnson PR.. Fowler JM. Laparoscopy versus laparotomy: an evaluation of adhesion formation after pelvic and paraaortic lymphadenectomy in a porcine model. Am J Obstet Gynecol 1998; 178: 499-503.

5. Liu CY. Laparoscopy hysterectomy, a review of 72 cases. I Reprod Med 1992; 37: 351-354.
6. Minelli L.. Angiolillo M.. Carione C.. Palamara V. Laparoscopically assisted vaginal hysterectomy. Endoscopy 1991; 23 :64-66.

7. Recio F.. Piver S.. Hempling RE. Pretreatment transperitoneal laparoscopic staging pelvic and paraaortic lymphadenectomy in large $\left({ }^{3} 5 \mathrm{~cm}\right)$ Stage IB2 cervical carcinoma: Report of a Pilot study. Gynecologic Oncology 1996; 63: 333-336.

8. González MA. Evaluación ex-ante de inversión en salud. El Hospital (USA) 1995; 51: 40-42.

9. Meeks GR., Harris RI. Surgical approach to hysterectomy: Abdominal, laparoscopy-Assisted, or vaginal. Clinical obstetrics and Gynecology 1997; 40: 886-894.

10. Ellström M., Ferraz-Nunes, Hahlin M.. Olsson J-H. A randomized trial with a cost-consequence analysis after laparoscopic and Abdominal Hysterectomy. Obstet Gynecol. 1998; 91: 30-34.

11. Spirtos NM.. Schlaerth JB., Kimball RE., Leiphart VM., Ballon SC. Laparoscopic radical hysterectomy type III with aortic and 
pelvic lymphadenectomy. Am J Obstet Gynecol 1996; 174: 17631768.

12. Hsieh YY., Lin W-C., Chang C-C., Yeh L-S., Hsu-T-Y.. Tsai H-D. Laparoscopic Radical Hysterectomy with low Paraaortic, Subaortic and pelvic lymphadenectomy. J Reprod Med 1998; 43: 528-534.

13. Schneider A., Possover M., Kamprath S., Endisch U., Krause N., Nöschel H. Laparoscopy-assisted radical vaginal hysterectomy modified according to Schauta-Stoeckel. Obstet Gynecol 1996; 88: $1057-1060$.

14. Roy M., Plante M., Renaud MC., Tetu B. Vaginal radical Hysterectomy versus abdominal radical hysterectomy in the treatment of early -stage cervical cancer. Gynecologic Oncology 1996; 62: 336-339.

15. Averette HE., Ravel R. Lymphography with chlorophyll: effects on pelvic lymphadenectomy and lymph nodes. Obstet Gynecol 1968; 37: 247-264.
16. Spirtos NM., Schaerth JB., Spirtos TW., Schaerth AC. et al Laparoscopic bilateral pelvic and paraaortic lymph node sampling: An envolving technique. Am J Obstet Gynecol 1995; 173: 105111 .

17. Meikle SF., Nugent LW., Orleans M. Complications and recovery from laparoscopy-assisted vaginal hysterectomy compared with abdominal and vaginal hysterectomy. Obstet Gynecol 1997: 89: 304-311.

18. Pastner B., Damien M. Umbilical metastases from a stage IB cervical cancer after laparoscopy: A case report. Fertil Steril 1992; 58: $1248-1249$

19. Wang P-H., Yuan CH-CH., Chao K-CH., Yen M-S., Ng H-T., Chao $\mathrm{H}-\mathrm{T}$. Squamous cell carcinoma of the cervix after laparoscopic surgery. J Reprod Med 1997; 42: 801-804.

\section{IMPORTANTE \\ LA REFERENCIA BIBLIOGRAFICA DE LA REVISTA COLOMBIANA DE OBSTETRICIA Y GINECOLOGIA ES:}

Rev Colomb Obstet Ginecol

DEBE CITARSE DE ESTA MANERA, YA QUE CORRESPONDE A LA NORMATIZACION Y RECONOCIMIENTO DE LA PUBLICACION A NIVEL INTERNACIONAL. 